SUPPLEMENTARY MATERIAL

\title{
TIME-WARPED GROWTH PROCESSES, WITH APPLICATIONS TO THE MODELING OF BOOM-BUST CYCLES IN HOUSE PRICES
}

Jie Peng, Debashis Paul and Hans-Georg Müller

Department of Statistics, University of California, Davis, CA 95616, USA 
In this supplementary material, we report a previous analysis based on a different fitting interval for estimating the growth rates $\alpha_{i}$. Specifically, we used the period between January 1998 and December 1999 to estimate the $\alpha_{i}$. This period was chosen based on the observation that for most areas the housing price followed a nearly exponential growth in the late nineties, as well as for convenience - the price indices are normalized to have the same value on January, 2000. In addition, in this analysis, we only used the price index data up to August 2012. So we treated January 1998 as time 0 and August 2012 as time 1 in the normalized time scale. We also set December 1999 as $t_{0}$ and used $\left[0, t_{0}\right]$ to estimate the $\alpha_{i}$ by equation (8) of the main text. The mean growth rate is $0.63 \%$ per month with a standard deviation $0.26 \%$ per month.

We then applied FPCA for the warping functions as described in Section 2 of the main text. The first two principal components explain $\sim 97 \%$ of the total variation in the warping functions. However, Las Vegas appears to be an outlier with a much larger second principal component than those of other areas. In order to avoid the influence of this outlier on our analysis, we dropped Las Vegas and re-did FPCA on the remaining 18 areas. Again, the first two principal components explain nearly $97 \%$ of the total variation (1st PC: $88.8 \%$, 2nd PC 7.7\%). Figure S-1 shows the first two eigenfunctions which display the same features as Figure 5 of the main text. In short, the first eigenfunction has a similar shape as the mean function which means a large fraction of the variance is explained by what amounts roughly to the degree at which the average boom cycle is expressed. Moreover, the first principal component primarily reflects the boom part of the cycle and the second component primarily reflects the bust part of the cycle. The second eigenfunction also went from positive to negative around 2008, which coincides with the onset of the economic recession. 
Figure S-1: First two eigenfunctions (multiplied by the square-root of their respective eigenvalues) of the time warping process
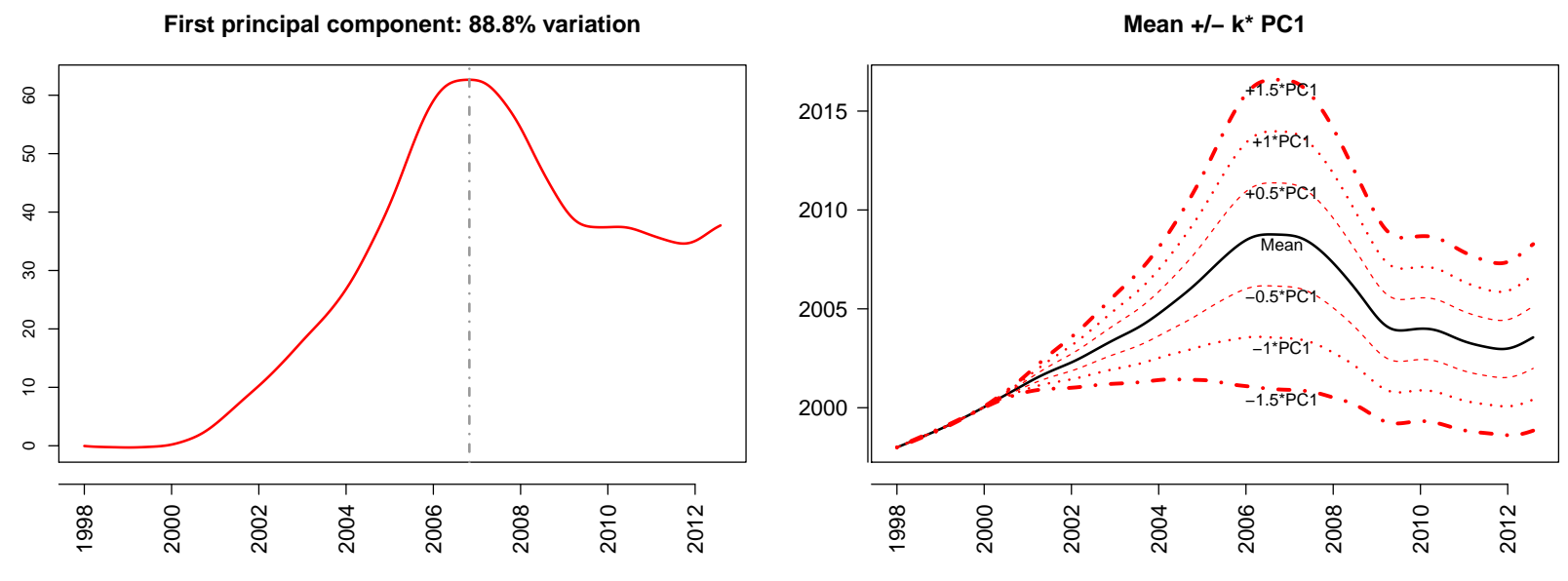

Second principal component: $7.7 \%$ variation
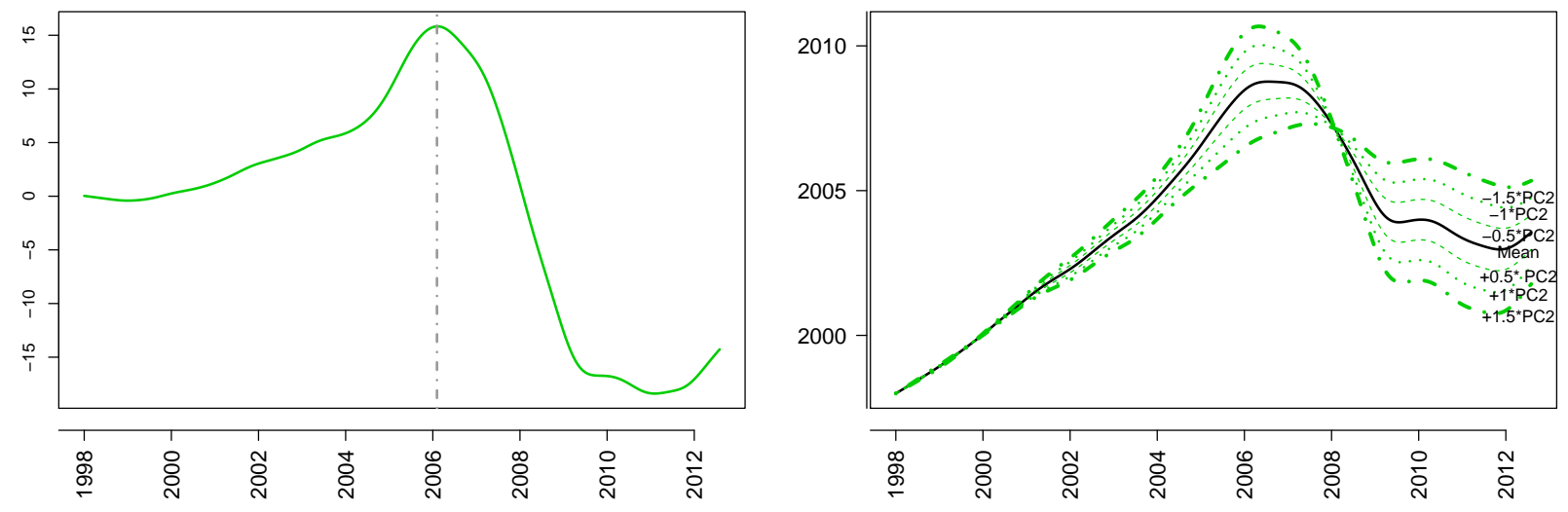